\title{
2. CIUDADES
}

\section{LA CIUDAD EN AMÉRICA LATINA: ENTRE LA GLOBALIZACION Y LA CRISIS}

\section{Fernando Díaz Orueta*}

Desde la primera mitad de los años ochenta, una serie de investigaciones vienen abordando el impacto producido por la globalización económica sobre los procesos de urbanización. En conjunto, dichos estudios han dado lugar a la constitución de un amplio marco de análisis, imprescindible para comprender las transformaciones aceleradas y trascendentales que afectan a las ciudades de todo el planeta ${ }^{1}$. Este artículo comienza recogiendo de forma resumida los aspectos fundamentales de la relación entre globalización y procesos de urbanización durante las dos últimas décadas. Más adelante, se pasa a analizar el impacto de la reestructuración económica sobre el sistema de ciudades latinoamericano. Este último está experimentando algunas variaciones significativas. Sin embargo, los graves problemas urbanos en las ciudades latinoamericanas no han hecho sino agudizarse durante esta etapa. Y, en gran medida, ello ha sido precisamente consecuencia de uno de los exponentes más claros de la reorganización económica en América Latina: las políticas de ajuste estructural.

\section{RESULTADOS DE LAS INVESTIGACIONES SOBRE LA CIUDAD GLOBAL}

Desde hace algún tiempo, se ha convertido en un lugar común el resaltar las particularidades del reciente proceso de internacionalización de la economía. En realidad, este no sería estrictamente novedoso puesto que, en realidad, desde hace varios siglos es posible hablar de una economía mundial. Sin embargo, tal y como han indicado diversos autores, la situación actual es notablemente diferente (Sassen, 1994). La creciente consolidación de espacios transnacionales de actividad económica, en los que los gobiernos comprueban como su papel disminuye día a día, ha hecho cambiar notablemente las condiciones en que tienen lugar la producción, el consumo y el intercambio de bienes y servicios ${ }^{2}$.

Estas transformaciones han provocado una evidente reorganización geográfica en la economía mundial. Sassen, tanto en el libro citado como en otros publicados anteriormente (Sassen, 1991), considera el incremento en la movilidad del capital y el paso paulatino hacia una economía de servicios, como elementos indispensables para comprender dicha reordenación. Durante las dos últimas décadas, una serie de grandes ciudades se habrían convertido en los lugares idóneos para dar cabida a las actividades y funciones ligadas a esa nueva forma de internacionalización de la economía. En ellas, la concentración y acumulación del capital internacional encuentran su "hábitat" natural. Además, las llamadas ciudades globales son los espacios adecuados para reunir los servicios avanzados y las telecomunicaciones, indispensables en el desarrollo y control de las operaciones económicas globales.

La terciarización, como fenómeno común a todos estos grandes centros urbanos, merece una especial atención. Siguiendo a Hall (1996), varias causas ayudarían a explicar su expansión. La más obvia sería que el crecimiento de las ganancias de productividad ha sido más veloz en el sector industrial. Un porcentaje no desdeñable de esta actividad

* Profesor Titular de Sociología Urbana en la Universidad de Alicante.

1. Seguramente, una de las evaluaciones más exhaustivas de los resultados alcanzados en la investigación sobre la ciudad global, sea la elaborada por Friedmann (1993). Tras sintetizar los conceptos básicos de esta serie de estudios y los debates teóricos subyacentes, Friedmann revisa dos de los trabajos comparativos más interesantes realizados sobre las ciudades globales, reseñando posteriormente el contenido de algunos estudios de caso.

2. De entre la bibliografía existente en español sobre la ciudad global, debe destacarse el monográfico publicado por la revista Alfoz en 1992. 
se ha desplazado a regiones periféricas desde sus antiguas localizaciones en los países desarrollados. Por otra parte, la utilización creciente de las nuevas tecnologías de la información ha contribuido a la terciarización de una forma definitiva. Dichas tecnologías estarían permitiendo una gran descentralización de los procesos productivos y haciendo realidad a muy bajo costo el movimiento casi instantáneo de información entre cualquier lugar del globo. La información se ha convertido en algo tan esencial que su producción e intercambio se ha consolidado como una actividad en sí misma ${ }^{3}$.

Ahora bien, el creciente peso específico del sector servicios debe entenderse también como consecuencia del aumento de una serie de ocupaciones ligadas de forma muy particular a esa expansión del terciario avanzado. Servicios de limpieza, de comida rápida, de hostelería, nuevos centros comerciales, etc, prestados a cambio de muy bajos salarios y en largas jornadas laborales. En suma, trabajadores imprescindibles para el mantenimiento y la reproducción de esta economía de servicios y, por lo tanto, muy abundantes en las grandes ciudades globales. Resulta también habitual que en estas ciudades una parte importante de los trabajadores mencionados sean inmigrantes extranjeros. Además, no puede olvidarse que este proceso tiene lugar en un momento de aplicación de políticas económicas impulsoras de forma genérica de la desregulación, las privatizaciones y el desmantelamiento del Estado de Bienestar.

De entre los países de Europa Occidental, seguramente sea en el Reino Unido donde dicha lógica se haya llevado más lejos. Por ejemplo, a propósito de las consecuencias que este proceso de reestructuración está teniendo sobre la economía de Cambridge (sureste de Inglaterra), una de las ciudades habitualmente citadas como lugar de crecimiento ligado al desarrollo de la nueva economía de servicios avanzados, el análisis realizado por Massey y Henry (1992) resulta muy esclarecedor. En lo que se refiere concretamente al mercado de trabajo, dichos autores concluyen que el crecimiento económico se está produciendo a costa de convertirse en uno de los espacios con mayores niveles de desigualdad de todo el país. De hecho, si se toma como un conjunto la región sureste de Inglaterra, la de mayor crecimiento económico en el Reino Unido en los años del experimento neoliberal, se observa que es precisamente allí donde las desigualdades de renta han aumentado de forma más acusada ${ }^{4}$.

Precisamente Sassen (1991), tras comparar la trayectoria seguida por Nueva York, Londres y Tokyo, concluye que es posible hablar de un incremento en las desigualdades en la distribución de los ingresos, del crecimiento de la pobreza y, a la vez, del aumento de la inversión local y extranjera en la construcción comercial y residencial de lujo. Como rasgos comunes más característicos deben mencionarse:

a) La "gentrification" que, aunque en realidad no es un proceso nuevo, sin embargo si presenta nuevos matices. En todas estas ciudades se han creado nuevos centros comerciales ligados al diseño y a la moda y espectaculares complejos para los negocios y las finanzas internacionales. En conjunto, estos proyectos, en muchas ocasiones junto con operaciones residenciales de lujo, actuan sobre las áreas centrales apropiándose de forma masiva de recursos públicos y espacio urbano.

b) Los mercados de trabajo temporales han terminado por institucionalizarse, pasando a ser la modalidad mayoritaria en las relaciones laborales de las economías de servicios.

c) En cuanto a los inmigrantes, estos presentan una distribución del empleo y de las ganancias claramente diferenciada de la de los "nativos".

\section{El sistema mundial de ciudades}

Las tendencias a las que se viene haciendo referencia han sido observados preferentemente en el grupo de grandes ciudades globales. Sin embargo, las transformaciones no se han manifestado únicamente en ellas. De hecho, la reorganización es de tal calibre que finalmente termina por afectar, de una u otra forma, a los distintos sistemas y subsistemas de ciudades. Uno de los intentos más rigurosos de valoración de estos cambios fue el realizado por Friedmann (1986). Tras sintetizar los principales contenidos de la aplicación del enfoque de la globalización al espacio urbano, este autor comienza por destacar como ciertas ciudades en el mundo estaban siendo utilizados por el capital internacional como auténticas bases de operación en la articulación y organización espacial de la producción y los mercados. El análisis de su estructura productiva y del empleo, dejaría a la luz una serie de especificidades que evidencian lo afirmado. Sin embargo, sus funciones no se limitarían a las estrictamente económicas. Así, también el control ideológico y de la comunicación se vienen ejerciendo, fundamentalmente, a través de las diversas informaciones, formas de ocio, etc, generadas en estas ciudades. Nuevos valores dominantes identificables con los sustentados por esa nueva clase social articulada también a escala global y caracterizada, a diferencia de la burguesía tradicional, por su no identificación estricta con un territorio concretos.

Una vez puestas estas bases, Friedmann analiza el actual sistema mundial de ciudades. En la cúpula se ubicarían ese escaso número de ciudades globales a las que se viene haciendo referencia. A partir de ellas, hacia abajo, aparece un sistema de ciudades marcado por la competitividad, la jerarquización y muy inestable. Tanto dentro de

3. Castells (1989) considera que es posible hablar de una auténtica revolución tecnológica. Su papel en la actual reestructuración del capitalismo resultaría esencial, considerando que las transformaciones son tan profundas que estarían modificando los propios conceptos de espacio y lugar. Unos años más tarde, Castells y Hall publicaron conjuntamente un libro muy esclarecedor respecto al surgimiento de una serie de nuevos espacios productivos y el papel que en ellos cumplen las nuevas tecnologías (Castells y Hall, 1994).

4. En España, Madrid es, sin duda, una de las áreas metropolitanas donde el intento de adaptación a la nueva situación resulta más claro. Sobre las consecuencias sociales y territoriales del proceso de reestructuración económica en Madrid, consultar: Díaz Orueta, 1992.

5. Sobre la aparición de una nueva clase capitalista transnacional: Sklair, 1991. 
los países centrales como en los semiperiféricos, el autor distingue ciudades primarias y secundarias. El resultado final es el siguiente ${ }^{6}$ :

\section{Países centrales}

- Primarias: Londres (I), Nueva York (I), Tokyo (I), Los Angeles (I), París (II), Chicago (II), Rotterdam (III), Frankfurt (III) y Zurich (III).

- Secundarias: Bruselas (III), Milán (III), Viena (III), Toronto (III), Madrid (III), Sydney (III), Miami (III), Houston (III) y San Francisco (III).

\section{Países semiperiféricos}

- Primarias: Sao Paulo (I) y Singapur (III).

- Secundarias: Buenos Aires (I), Río de Janeiro (I), México (I), Hong Kong (II), Manila (II), Bangkok (II), Seul (II), Johanesburgo (III), Caracas (III) y Taipei (III).

Del mismo modo que las principales ciudades globales cumplirían papeles articuladores de la economía a escala mundial, otras lo harían a escala regional, nacional o internacional. A juicio de Friedmann, en numerosas ocasiones resulta complejo situar exactamente el lugar que ocupa en esa jerarquía una determinada ciudad. A la gran inestabilidad del sistema mundial de ciudades, hay que añadir la ausencia de un criterio universal que sirva para enmarcar exactamente cada situación ${ }^{7}$. La revisión de la abundante bibliografía al respecto deja a la luz significativas diferencias en las jerarquías establecidas por los diversos autores.

De hecho, en un trabajo publicado recientemente, Short, Kim, Kuus y Wells (1996) destacan la existencia de grandes lagunas en la información comparativa manejada habitualmente en la investigación sobre las ciudades globales. Fuera de los datos demográficos básicos suministrados por el Demographic Yearbook de las Naciones Unidas, existen muy pocas oportunidades de encontrar información comparativa de calidad. El problema se complica, y mucho, en la recopilación de información sobre aspectos trascendentales: los grandes centros financieros, las sedes de las mayores corporaciones, los principales nodos de telecomunicaciones, los centros de transporte y los lugares de celebración de grandes espectáculos de resonancia mundial. Tras un exhaustivo análisis de la bibliografía sobre la investigación urbana en esta materia, los autores concluyen que se ha reforzado la supremacía de Tokyo y, en menor medida de París. A la vez, detectan un cierto declive en Londres y Nueva York y la emergencia en un escalón secundario de ciudades como Frankfurt y Seul.

Por último, antes de entrar a analizar específicamente las ciudades latinoamericanas, es preciso detenerse en un término que recobraremos más adelante. Se trata del concepto "máquina de crecimiento" (growth machine en inglés). En las ciudades, especialmente en aquellas sometidas a un mayor crecimiento, se produce históricamente una fuerte competición alrededor de los usos que se pretende dar al espacio. Detrás del crecimiento de las ciudades están las que Logan y Molotoch (1987) denominaron coaliciones por el crecimiento. Las mismas están formadas por aquellos grupos que trabajan conjuntamente por favorecer el crecimiento en una determinada ciudad ${ }^{8}$. De la correlación de fuerzas sociales, políticas y económicas, depende la orientación dada al crecimiento de cada ciudad. Ciertos espacios resultan los más disputados en función de sus características específicas. En ocasiones, los movimientos urbanos populares consiguen hacer prevalecer aquellos usos favorables a los sectores más necesitados, pero cuando las coaliciones por el crecimiento son poderosas sus decisiones se convertirán en prácticamente incuestionables.

Durante los últimos años, la idea de la competitividad ha permeado de forma muy acusada los discursos sobre el territorio. En virtud de ella, se han establecido luchas despiadadas por atraer grandes inversiones, se han realizado enormes esfuerzos para construir costosas infraestructuras, se han gastado notables sumas de dinero en definir estrategias que determinasen el lugar a ocupar dentro del sistema mundial de ciudades,...Y en numerosas ocasiones, todo ello se ha llevado a cabo a costa del desvío de fondos de partidas destinadas a otros fines. Por lo tanto, en la actualidad las máquinas por el crecimiento encuentran un marco de actuación muy favorable. Plantear dudas respecto a las decisiones de las coaliciones locales por el crecimiento lleva a convertirse en destinatario de las críticas más contumaces, puesto que supuestamente se estarían cuestionando propuestas trascendentales e inamovibles, basadas en una subordinación absoluta de lo local a lo global ${ }^{9}$. Más adelante volveremos sobre los enormes e inútiles esfuerzos a los que puede llevar en algunas ciudades latinoamericanas optar acríticamente por un discurso de este tipo.

\section{LAS CIUDADES LATINOAMERICANAS EN EL CONTEXTO DE LA GLOBALIZACION}

En primer lugar, y como resulta casi obvio recordar en un artículo de estas características, sería inexacto referirse a procesos urbanos completamente generalizables al con-

6. La clasificación de las ciudades (regiones metropolitanas) según el tamaño de su población queda definida como sigue: I (entre 10 y 20 millones de habitantes); II (entre 5 y 10) y III (entre 1 y 5 millones). Los criterios de selección utilizados por el autor fueron: consideración o no como grandes centros financieros, sedes centrales de transnacionales, presencia de instituciones internacionales, crecimiento acelerado del sector servicios avanzado, principales centros de comunicación y tamaño demográfico. No fueron utilizados todos los criterios en todos los casos, pero si resultó necesario satisfacer varios criterios para poder considerar a una ciudad como global de un determinado rango. A pesar de que habría sido posible añadir terceros o incluso cuartos órdenes en la jerarquía establecida, se optó por no hacerlo debido a que el principal interés del autor consistía en identificar únicamente los centros de acumulación capitalista más importantes.

7. De hecho en el artículo de 1993 , donde Friedmann evaluaba la investigación sobre las ciudades globales en la década anterior, el autor introdujo algunas nuevas que, a pesar de no aparecer en el listado elaborado en 1986, sin embargo habrían ido adquiriendo una notable relevancia. Así ocurrió con Shangai, Osaka, Vancuver y Seattle o Dusseldorf y Munich.

8. En ellas intervienen políticos, líderes de opinión de los medios de comunicación locales, promotores inmobiliarios, universidades, sindicatos, profesionales liberales, comerciantes detallistas, grandes empresarios e, incluso, los equipos deportivos profesionales de la localidad.

9. Por ejemplo en España así ocurrió con los grupos que se atrevieron a criticar, aun cuando fuera parcialmente, algunas de las decisiones tomadas en materia urbana al calor de la EXPO-92 de Sevilla o de los Juegos Olímpicos de Barcelona. 
junto de América Latina. Al contrario, entre unas y otras ciudades existen diferencias profundas en cuanto a los tiempos, ritmos y formas de urbanización. La evolución histórica de cada uno de los países de la región, ha condicionado económica, política y socialmente los procesos urbanizadores. Además, por si ello fuera poco, en numerosos países es posible encontrar importantes variaciones internas que han dado lugar a modelos de desarrollo urbano muy diferenciados. Por lo tanto, más allá del necesario esfuerzo de generalización que se llevará a cabo a continuación, debe recordarse que cada ciudad, cada área metropolitana, presenta especificidades que tienen que ser tenidas en cuenta al abordar su análisis. Ahora bien, ello no resulta contradictorio con preguntarse por la forma en que el proceso de globalización descrito ha podido afectar a las ciudades latinoamericanas. Nos situaríamos así en un enfoque similar al planteado por algunos investigadores urbanos de la región (Carrión, 1987, Lungo, 1995).

\section{Reestructuración productiva en América Latina: los efectos de la globalización}

La crisis económica mundial de los años setenta tuvo importantes consecuencias sobre las economías latinoamericanas. A mediados de esa década, se produjo una gran recesión en los países centrales que se cebó en sectores industriales estratégicos (siderurgia, naval, etc). En realidad, la reorganización de la economía mundial de la que venimos hablando en este artículo, encontró su discurso justificativo en la necesidad de superar dicha situación de estancamiento. Pradilla (1989) resumió con acierto los impactos de esta crisis sobre las economías latinoamericanas: el parón en el patrón de industrialización por sustitución de importaciones, la internacionalización del capital, la reconcentración monopólica en todas las áreas de la actividad económica, la agudización de la crisis agrícola, el enorme endeudamiento privado y público con la Banca multinacional y local, el crecimiento de las importaciones con un alto componente de los medios de consumo suntuario e inmediato, una gran fuga de capitales hacia los países centrales, el aumento en el déficit en la balanza de pagos y el crecimiento de los procesos inflacionarios.

En gran medida, el capitalismo central superó la crisis trasladándolo al periférico. En ese "traslado", los mecanismos de crédito ocuparon un papel trascendental. La participación de los organismos internacionales vigilantes de la orientación de las políticas económicas (fundamentalmente, el Fondo Monetario Internacional y el Banco Mundial), resultó esencial. Siguiendo sus orientaciones América Latina llegó a convertirse en exportadora neta de capitales ${ }^{10}$.

Las políticas de ajuste estructural pasaron a ser entonces la receta aplicada de forma generalizada a las economías latinoamericanas. Los países centrales, el Banco Mundial y el Fondo Monetario Internacional fueron los encargados de vigilar el proceso. En realidad, estas políticas han marcado la pauta de la integración de las economías de los distintos países latinoamericanos en el proceso de globalización. Las líneas de intervención han sido las siguientes (Pradilla, 1989): a) La privatización de las empresas estatales, dejando este sector reducido a aquellas ramas sin interés para el capital privado.

b) La penetración del capital privado, local y extranjero.

c) La reducción drástica del gasto público en el sector social.

d) La austeridad salarial, con reducciones tanto en el salario directo como en el indirecto.

e) El desmantelamiento de las conquistas de los trabajadores.

Todo ello se ha ido llevando a cabo a la vez que se articulaba un nuevo modelo exportador, fundamentado en la introducción de productos agrícolas o agroindustriales no tradicionales, las zonas francas y el turismo. Detrás de esta política económica hay una sólida apuesta ideológica, que coincide en algunos de los principales países centrales (Reino Unido y Estados Unidos especialmente) con un momento de exaltación del pensamiento neoliberal. Recuperando las posiciones más dogmáticas del antiguo liberalismo, se insiste en la necesidad de disminuir a cualquier coste el tamaño del Estado, afirmando que, de este modo, el mercado actuaría por si mismo "aprovechando las mejoras comparativas de cada país". Los resultados también hablan por si mismos.

\section{Sistema de ciudades y globalización}

En primer lugar, es preciso describir brevemente las principales características del sistema de ciudades latinoamericano. Diversos estudios vienen señalando que América Latina en su conjunto se convertirá en el año 2000 en la región más urbanizada del planeta, fuera del mundo desarrollado. La información recogida por Valladares y Prates Coelho (1996) resulta útil para tomar conciencia de las diferencias en los niveles de urbanización de la región:

a) Un primer grupo de países presenta tasas de urbanización superiores al 70\%. Entre ellos se encuentran algunos de los que iniciaron su proceso de urbanización masivo en las primeras décadas de este siglo (Argentina, Uruguay y Chile) y otros que conocieron un fuerte crecimiento urbano a partir de los años cuarenta (Venezuela, Brasil, México y Perú).

b) Países con una urbanización entre el $50 \%$ y el $70 \%$ : Colombia, Ecuador, Cuba, Nicaragua, Panamá y Bolivia.

c) Por debajo del $50 \%$ se encuentra el resto de los países centroamericanos.

10. Merino del Río escribía en 1989:

"Es en torno a este problema donde más cruda y dramáticamente se reflejan las contradicciones del sistema capitalista mundial: los polos de pros peridad y despilfarro en los centros imperialistas (Estados Unidos, Japón, Europa Occidental) alimentan su insolente riqueza sobre la base de unos intereses de la deuda que bace crecer, en el otro polo, la miseria de los tugurios, favelas, chabolas, villas miseria, cantegriles, callampas" (Merino del Río, 1989:79). 
En cuanto a la organización del sistema de ciudades latinoamericano existe un elevado grado de acuerdo respecto a su caracterización ${ }^{11}$. Se recupera ahora la clasificación establecida por Borja, Castells y Belil (1989):

a) Existe un número reducido de megalópolis, por encima de los diez millones de habitantes: México, Sao Paulo, Buenos Aires y Río de Janeiro ${ }^{12}$. Aunque con variaciones, en general sus ritmos de crecimientos se han moderado durante los últimos años, lo que no significa que sus problemas urbanos se encuentren en vías de solución.

b) En segundo lugar, aparecen una serie de ciudades entre tres y siete millones de habitantes. Con las tasas de crecimiento actuales, su tamaño alcanzará en poco tiempo enormes dimensiones. Algunos ejemplos son: Bogotá, Lima, Santiago y Caracas.

c) Las ciudades pequeñas y medianas han aumentado de forma destacable su peso específico. Alguno de estos núcleos urbanos presenta tasas muy acusadas de incremento demográfico. Como ejemplo, se pueden citar las ciudades de Salvador de Bahía, Belo Horizonte, Guayaquil y Medellín.

d) En último lugar, se encuentran los asentamientos rurales, poblaciones de menos de 2000 habitantes, donde todavía reside una parte significativa de la población latinoamericana.

En numerosas áreas de América Latina, la expansión de las zonas francas y de la actividad turística a gran escala ha favorecido la pérdida de peso relativo de las mayores ciudades. Sin embargo, en los años más recientes también se detectan síntomas que parecen señalar un posible reforzamiento de los mayores núcleos urbanos.

Concretamente, Portes (1988) ha destacado como uno de los rasgos más novedosos de la reciente evolución del sistema urbano latinoamericano, la desaceleración de la primacía urbana ${ }^{13}$. La agudización del crecimiento demográfico de las ciudades secundarias se ha convertido en un fenómeno lo suficientemente extendido como para preguntarse seriamente por sus causas. El autor centra fundamentalmente su explicación en las transformaciones aparecidas en las estructuras productivas, tras la crisis del modelo de industrialización por sustitución de importaciones. El paso hacia un modelo económico centrado en las exportaciones ha hecho que la crisis de la industria tradicionalmente localizada en las grandes ciudades, haya venido acompañada de la aparición de nuevas empresas en otras localizaciones. Portes pone como ejemplo el desarrollo de las zonas francas ${ }^{14}$.

De todos modos, seguramente todavía es pronto para conocer en profundidad hasta donde llegará esta tendencia $^{15}$. Al preguntarse por lo sucedido en Centroamérica y el Caribe, Lungo y Portes (1992) señalan que la pérdida de ingresos y las mayores dificultades para conseguir un empleo, como consecuencia de la reestructuración económica, son factores que continuan empujando a la población hacia las grandes ciudades, buscando las oportunidades que esta ha ofrecido tradicionalmente. Pero, a la vez, dichos autores piensan que esta misma tendencia se ve contrarrestada por otras tres: a) el agotamiento de las expectativas económicas en las ciudades, b) la migración internacional, a la búsqueda de aquello que estos ciudadanos no pueden encontrar en sus países de origen y c) la nueva atracción surgida como consecuencia de las zonas francas y el turismo ${ }^{16}$.

Ahora bien, la propia Sassen (1994) ha señalado algunos indicios que irían, sin embargo, en la línea de profundizar el peso específico de las grandes ciudades latinoamericanas. Desde 1991 tiene lugar un significativo crecimiento de las inversiones extranjeras directas en la región, fortaleciendo el papel de las mayores ciudades como centros de negocios. Así estaría ocurriendo, por ejemplo, en México, Sao Paulo y Buenos Aires. Los grandes procesos privatizadores, junto con la desregulación de los mercados financieros llevada adelante en estos países, se encontrarían en la base de este aluvión de inversión extranjera. Siempre siguiendo a Sassen, en estas tres ciudades aparecen ya algunos procesos evidentes en muchas de las grandes ciudades occidentales ${ }^{17}$ :

11. Sobre esta materia, debe reseñarse un interesante artículo de Hardoy (1993) sobre el futuro de la ciudad latinoamericana. En él, tras explorar demográficamente desde un punto de vista cuantitativo las dimensiones actuales de las ciudades latinoamericanas, Hardoy realiza un valioso ejercicio prospectivo. El autor termina planteando una serie de iniciativas, todas ellas orientadas a la consecución de una mejor ciudad para el futuro.

12. Las ciudades de este primer grupo son precisamente las únicas latinoamericanas, además de Caracas, incluidas por Friedmann (1986) en el listado ya citado. En la escala, Sao Paulo era considerada una ciudad de tipo primario. Buenos Aires, Río de Janeiro y México quedaban incluidas entre las ciudades secundarias. Todas ellas pertenecientes en la clasificación de Friedmann a países semiperiféricos.

13. Concentración en la mayor ciudad de un país de una gran parte de la población y la actividad económica.

14. Según Sassen (1994), es esta una de las formas de expresión de la recomposición geográfica favorecida por la globalización económica. Se trataría de espacios ubicados en países con bajos salarios, donde algunas firmas de las naciones más desarrolladas deciden localizar sus fábricas con el objetivo de ensamblar o procesar ciertos componentes, posteriormente reexportados a los países centrales. Se cuenta con una legislación especial que ampara esta situación. Las zonas francas se han convertido en un mecanismo clave en la internacionalización de la producción.

15. Incluso algunos autores han matizado su importancia. Por ejemplo, Jaramillo y Cuervo (1990) explican los cambios en las primacías urbanas, más por un componente de mecánica demográfica que por la reacción frente a la crisis. De hecho, “(...) O certo é que o que mais sobres sai nessa época de crise para os países da regiao é a continuidade nas hierarquias das redes urbanas e a manutençao de seus padroes regionais. Nao parecem estar presentes as reestruturaçoes nítidas que podem ser observadas nos países centrais" (Jaramillo y Cuervo, 1990:115). Los autores citados destacan tres factores como los fundamentales para explicar las diferencias entre los países centrales y los latinoamericanos: a) el diferente papel jugado por la innovación tecnológica en una y otra área, b) la mayor heterogeneidad espacial en cuanto a las condiciones de acumulación en Latinoamérica, con la necesidad de concentrar los grandes equipamientos y las inversiones públicas y privadas en puntos muy localizados del territorio, y c) la escasa capacidad de resistencia de los trabajadores a la reorganización productiva, que mitigaría la necesidad de la descentralización industrial por parte del capital.

16. La proliferación de centros turísticos destinados al ocio de los ciudadanos procedentes de los países desarrollados, ha crecido de forma espectacular. Ello está llevando a la formación de auténticas reservas destinadas al esparcimiento de estos "ciudadanos de primera clase", alejados de la miseria en la que se mueve una parte muy importante de la población "autóctona".

17. En los próximos años deberá valorarse en qué medida estas tendencias pueden terminar dando lugar a fenómenos que vayan materializando nuevas geografías sociales, al estilo de lo ocurrido por ejemplo en las principales ciudades globales con los procesos de "gentrification". 
a) Unos mercados financieros muy dinámicos, acompañados de la aparición de sectores de servicios especializados.

b) La sobrevaloración del output, firmas y trabajadores pertenecientes a estos sectores.

c) Simultáneamente, la desvalorización del resto del sistema económico.

Por lo tanto, en los últimos años se vienen manifestando una serie de nuevas tendencias, en ocasiones orientadas en sentidos aparentemente opuestos ${ }^{18}$. La especificidad de algunos de los cambios sucedidos en las ciudades y regiones latinoamericanas, es consecuencia de la forma particular en que el proceso globalizador las afecta. En realidad, la no coincidencia de determinados procesos de transformación en unos y otros escenarios, no hace sino expresar la naturaleza concreta de su inserción en la División Internacional del Trabajo.

\section{Ciudad, desigualdad y crisis social}

Pero si únicamente se tomasen en cuanta las variaciones en el territorio a gran escala, la consideración del impacto del proceso de globalización sobre las ciudades latinoamericanas quedaría incompleto. Las consecuencias sociales de las políticas de ajuste se han sufrido en las ciudades de una manera especial. Las estructuras sociales urbanas se encuentran inmersas en un proceso de cambio muy acusado. El número creciente de personas ocupadas en la economía informal, el enorme aumento de las tasas de desempleo, la pérdida constante y selectiva (más acusado en unos sectores que en otros) de poder adquisitivo y una mayor desigualdad en la distribución del ingreso, son cuatro de las principales consecuencias del proceso de "modernización excluyente" ${ }^{19}$. Como afirma González Villar:

\section{“(...) El modelo despliega múltiples contradicciones, generando una profunda polarización entre ocupacio- nes plenas y no plenas, entre salarios altos y salarios bajos, como oscuro telón de fondo de la transforma- ción productiva se fragmenta la sociedad, incremen- tándose los diferenciales de poder, constituidos y constituyentes de los procesos de desestructura- ción/reestructuración social. En esta situación las identidades sociales se diluyen. Segmentación, bete- rogeneización, polarización, atomización, fragmenta- ción son los signos que permiten interpretar la clave de la sociedad actual" (González Villar, 1995: 24).}

El descenso del gasto público en sectores básicos (vivienda, sanidad, educación, etc) ha repercutido notablemente en las condiciones de vida de amplias capas de la población ${ }^{20}$. En este nuevo tiempo, y siguiendo las recomendaciones neoliberales más ortodoxas, las políticas sociales dejan de tener como objetivo la búsqueda de una mayor igualdad social. Ahora es el tiempo de la aplicación de acciones selectivas, dirigidas únicamente a aliviar algunas de las situaciones más extremas. Así también ocurre en materia urbana, donde el Banco Mundial ha insistido en el carácter temporal de los problemas (Banco Mundial,
1995), renunciando explícitamente de este modo a asumir una postura que llevaría directamente a la necesidad de acometer cambios estructurales.

Como consecuencia, los problemas urbanos se han agravado notablemente. La vivienda, los equipamientos, las infraestructuras, el medio ambiente y, en definitiva, la propia gestión urbana en su conjunto, se han convertido en auténticas pesadillas para aquellos gobiernos municipales latinoamericanos empeñados en tratar de afrontar esta situación, pero sin contar con las competencias y los medios necesarios. En los núcleos urbanos pequeños y medianos sometidos a altas tasas de crecimiento demográfico, las posibilidades de favorecer una urbanización ordenada y previsora, que evite los problemas y desajustes sufridos en las grandes ciudades, son prácticamente nulas. Los asentamientos precarios, la ausencia de equipamientos e infraestructuras, el deterioro medioambiental, la ocupación de espacios peligrosos, etc, resultan aspectos recurrentes en la mayor parte de estas ciudades.

La pobreza afecta, en sus distintas manifestaciones, cada vez a mayores sectores de población. Sin embargo, y frente a lo ocurrido en otros momentos históricos, ello no parece estar dando lugar de forma generalizada a una creciente polarización socio-espacial. Diversos estudios realizados en los últimos años ${ }^{21}$, coinciden, en mayor o menor medida, en una descripción del espacio urbano donde la mayor proximidad en la localización residencial de las distintas clases sociales se debe a la propia precariedad y no a una sustancial mejora de las condiciones socio-económicas de la población. La profundidad de la crisis social obliga a grupos importantes de población a desplazarse a localizaciones centrales, buscando una mayor proximidad a sus posibles fuentes de trabajo y abaratando costes de transporte. A la vez, en muchas ciudades latinoamericanas sectores de altas rentas se han ido moviendo hacia las periferias, instalándose en auténticos enclaves fuertemente protegidos. En la mayoría de las ocasiones, el resultado final se asemeja al descrito por Caldeira (1996) para Sao Paulo: una ciudad claramente fragmentada, donde la cercanía física no es sinónimo de proximidad social. Un espacio heterogéneo pero roto. Muchas calles, anteriormente espacios de encuentro, juego y paseo, se han convertido en lugares vacíos, rodeados de muros, vallas y controlados por policías privados.

18. Por ejemplo, la tensión entre aquellos factores que empujan al crecimiento de los grandes centros urbanos y aquellos otros que tienden a moderarlo.

19. De esta forma tan elocuente, denominan Barbeito y Lo Vuolo (1992) la serie de transformaciones económicas y sociales en marcha en la Argentina durante los últimos años. En realidad, dicho término resulta perfectamente válido para aplicar a lo ocurrido en muchos otros países latinoamericanos.

20. Benería (1995) destaca la puesta en marcha de estrategias de supervivencia que tratan de afrontar el proceso de empobrecimiento masivo. Estas se habrían dirigido en tres direcciones: a) los ajustes en los mercados de trabajo, b) los ajustes del presupuesto familiar, y c) la reestructuración de la vida cotidiana. Por lo tanto, el peso del ajuste, además de tener un evidente sesgo clasista, lo tiene también de género, al haber recaido de una forma especial sobre las mujeres.

21. Por ejemplo: Portes (1988), Portes y Lungo (1992), Torres (1993), Urrutia (1994), Díaz Orueta (1994), Caldeira (1996), Lourés (1996). 
Las casas y edificios de apartamentos han quedado separados de la calle por numerosas medidas de seguridad. El paisaje urbano se transforma notablemente y lo hace en función de una nueva y triste estética de la seguridad. Estética también presente en los espacios terciarios (oficinas y shoppings).

En realidad, se trata de la expresión física de la creciente ingobernabilidad. ¿En qué medida continuará siendo posible hablar de una situación de normalidad democrática cuando sectores de la población, en muchas ocasiones mayoritarios, son excluidos económica y socialmente?. Algunos investigadores urbanos ya han comenzado a tratar de dar respuesta a una pregunta que, sin duda, deberá convertirse en fundamental durante los próximos años ${ }^{22}$.

\section{CONCLUSIONES}

Por lo tanto, la complejidad de los problemas y contradicciones a afrontar en las ciudades latinoamericanas es enorme. Desde luego, existen algunas tendencias de fondo que pueden permitir establecer ciertos paralelismos con lo ocurrido en los países centrales. Sin embargo, la magnitud de los problemas a abordar, las especificidades del proceso urbanizador latinoamericano y, sobre todo, el escaso margen de maniobra que deja el proyecto de adaptación a la globalización a través de la modernización excluyente, obligan a la realización de una reflexión propia. Una reflexión que, sin olvidar el contexto esbozado en las primeras páginas de este artículo, camine hacia la determinación de un marco de análisis específico que trascienda la crítica que se queda en el lamento pasivo por lo negativo de la situación o, en sentido contrario, en las infundadas previsiones del Banco Mundial, solicitando más paciencia puesto que "nunca llovió que no escampara".

El énfasis puesto en la necesidad de convertir también a las ciudades y metrópolis latinoamericanas en "competitivas", aprovechándose de sus "ventajas comparativas", resulta erroneo. La puesta en marcha de una estrategia que, incluso en muchas ciudades de los países centrales, está cosechando fracasos estrepitosos, sólo traerá beneficios para las coaliciones por el crecimiento de las localidades respectivas. En dicha estrategia se está encontrando la coartada perfecta para continuar retrasando el momento de afrontar la crisis urbana. Tal y como ya se percibe en distintos países latinoamericanos, y contrariamente a lo afirmado por los defensores de este modelo, por esta vía no va a haber reparto después del crecimiento.

El principal desafío para los próximos años es, utilizando palabras de Fernández Durán (1993), conseguir transformar la creciente ingobernabilidad en antagonismo. Existe un discurso económico y territorial dominante, vinculado a los intereses de la nueva clase capitalista transnacional, que es preciso dejar en evidencia, mostrando su fracaso y sus enormes contradicciones. La tarea no es sencilla, puesto que las fuerzas que lo sustentan son muy poderosas y controlan los principales medios de formación de masas. Además, a pesar del carácter estructural de la globalización, sin embargo también es cierto que cada ciudad, cada región, presenta su propia historia, una correlación de fuerzas políticas y sociales particular, que invalidaría cualquier tipo de planteamiento resolutivo universalista. Para terminar, unicamente querría plantear algún comentario respecto al papel a desarrollar por las ONGs, una de las formas de organización social más citadas cuando se abordan las posibles alternativas de solución al actual estado de cosas.

Desde luego, está fuera de dudas que muchas de estas organizaciones están aportando un trabajo importante en el intento de solventar los peores efectos de la crisis social descrita, tanto en el medio rural como en el urbano. Sin embargo, no parece tan evidente que en la mayor parte de los casos su labor de apoyo esté suponiendo un avance sustancial en ese intento de convertir la ingobernabilidad en antagonismo. No debe olvidarse que en los propios documentos del Banco Mundial aparece la recomendación de contar con las ONGs en amplias áreas de la gestión urbana. En realidad, como plantea Lungo (1992), esa idea es coherente con una corriente que desde finales de los años setenta viene defendiendo el cuestionamiento de la planificación urbana, las prácticas descentralizadoras como intrínsecamente positivas y la necesidad de llevar adelante políticas indiscriminadas de privatización de los servicios urbanos. A esta visión neoliberal, se le adapta como anillo al dedo la idea de dejar en manos de las ONGs labores de apoyo a la gestión urbana, especialmente en áreas populares. Una vez que el Estado ya no es considerado responsable último de la gestión urbana, se piensa que esta puede ser una buena solución. Lungo (1992), sin descalificar por completo este tipo de orientación, realiza dos objeciones fundamentales: a) supone la puesta en marcha en el ámbito urbano de numerosas acciones aisladas, sin un plan que las de sentido, y b) se atomizan las reivindicaciones de los sectores urbanos populares.

En la medida en que, habitualmente con las mejores intenciones, se reivindica el reforzamiento de estas organizaciones, sin entrar en mayores contenidos, se está consolidando también el nuevo rol del Estado: no intervencionista en lo social, desregulador y menos inversionista. Al no ser sustituido por ninguna otra instancia, el ya de por sî débil papel que cumplía en América Latina el Estado como orientador de la planificación urbana, se desvanece. La ciudad va quedando sometida a las libres fuerzas del mercado, que campan a sus anchas en un escenario donde el disenso se expresa de una forma desorganizada, fragmentada y sin una perspectiva de conjunto.

En definitiva, las ONGs, o al menos algunas de ellas, deberán jugar un papel importante en las alianzas de fuerzas a constituir en cada ciudad con el objetivo de hacer frente a las coaliciones por el crecimiento. Como aglutinante de todos estos grupos deberá existir un proyecto para el conjunto de la ciudad, superador de las tendencias actuales a la fragmentación y a la heterogeneidad. Por ello, es decisivo que cada grupo sectorial, cada área territorial sea capaz de articular sus intereses parciales dentro de un proyecto global ${ }^{23}$.

22. Sobre Brasil: Valladares y Prates Coelho, 1995.

23. En este sentido, resulta muy oportuna la lectura del texto escrito por Singer (1995) sobre los problemas planteados durante el gobierno del P'T en Sao Paulo. 


\section{BIBLIOGRAFIA}

Alfoz, N. ${ }^{\circ}$ 90, 1992.

Banco Mundial. (1995), Política urbana y desarrollo económico: un programa para el decenio de 1990, Washington: Banco Mundial.

Barbeito, Alberto C. y Lo Vuolo, Rubén M. (1992), La modernización excluyente, Buenos Aires: UNICEF/ LOSADA.

BENERIA, Lourdes. (1995), "Los costes sociales del ajuste estructural en América Latina. ¿Está superada la crisis?", Mientras Tanto, n. ${ }^{\circ} 61$.

Borja, Jordi, Castells, Manuel y Belil, Mireia. (1989), "Descentralización y gestión urbana", en: LUNGO, Mario., Lo urbano: teoría y métodos, San José de Costa Rica: CSUCA.

CALDEIRA, T.P.R. (1996), "Building up walls: the new pattern of spatial segregation in Sao Paulo", International Social Sciende Journal, n. ${ }^{\circ} 147$.

CARRIÓN, Fernando. (1987), La investigación urbana en América Latina: caminos recorridos y por recorrer, Quito: Ciudad.

CASTELlS, Manuel. (1989), The Informational City, Oxford: Blackwell Publishers.

Castells, Manuel y Hall, Peter. (1994), Las tecnópolis del mundo, Madrid: Alianza Editorial.

Cepal (1989), La crisis urbana en América Latina y el Caribe. Reflexiones sobre alternativas de solución, Santiago de Chile.

Díaz Orueta, Fernando. (1992), Desigualdades socioterritoriales en la Comunidad de Madrid en la etapa de reestructuración económica, Madrid: Editorial de la Universidad Complutense (Colección Tesis Doctorales).

Díaz Orueta, Fernando. (1994), "Buenos Aires: por las veredas del ajuste", América Latina Hoy, n. ${ }^{\circ} 7$.

FERNÁNDEZ DURÁN, Ramón. (1993), La explosión del desorden. La metrópoli como espacio de la crisis global, Madrid: Fundamentos.

FriedmanN, John. (1986), "The World City Hypothesis", Development and Change, Vol.17.

FriedmanN, John. (1993), "Where we stand: a decade of World City Research”, Paper presentado a la Conference of World Cities in a World System, Sterling.

Gilbert, A. y Gugler, J. (eds), Poverty and Development Urbanization in the Third World, Oxford: Oxford University Press.

GonZÁlez Villar, Carlos. (1995), "Desigualdad, exclusión y pobreza", Estudios Regionales, n. ${ }^{\circ} 8$.

Hall, Peter. (1996), "The Global City", International Social Science Journal, n. ${ }^{\circ} 147$.

HARDOY, Jorge Enrique. (1993), "El futuro de la ciudad latinoamericana", Medio Ambiente y Urbanización, n. ${ }^{\circ}$ 43-44.

JARAmillo, Samuel y Cuervo, Luis Mauricio. (1990), “Tendências recentes e principais mudanças na estrutura espacial dos países latino-americanos", en: VALLADARES, Licia y PRETECEILle, Edmond. (coords.), Reestruturaçao Urbana: Tendências e Desafios", Sao Paulo: Nobel.
LAURELLI, Elisa y Lindenboim, Javier. (1990), Reestructuración económica global. Efectos y políticas territoriales, Buenos Aires: CEUR.

Logan, John y MoLOTOCH, Harvey L. (1987), Urban Fortunes: The Political Economy of Place, Berkeley: University of California Press.

LOURES, María Luisa. (1996), Buenos Aires: centro bistórico y crisis social, Madrid: Universidad Complutense (Tesis Doctoral).

Lungo, Mario. (1989), Lo urbano: teoría y métodos, San José de Costa Rica: CSUCA.

Lungo, Mario. (1993), Procesos urbanos, San Salvador: Istmo.

Lungo, Mario. (1995), "América Latina hacia el final del siglo XX: ¿se está configurando un nuevo patrón de urbanización?”, en: DiAz Orueta, Fernando y Mira, Eduard., (eds), Pensar y Vivir la Ciudad, Alicante: Departamento de Ciencias Sociales.

Massey, Doreen. (1994), Space, place and gender, Cambridge: Polity Press.

MASSEY, Doreen y AlLEN, John, (1988), Uneven Re-Development. Cities and Regions in Transition, Londres: Hodder \& Stoughton.

Massey, Doreen y Henry, Nick. (1992), Sometbing new something old: a sketch of the Cambridge economy, The South East Programme, Occasional Paper Series, $\mathrm{n}^{\circ} 2$

Merino Del Río, J. (1989), "La deuda externa: pagar o vivir", en: VARIOS Autores, Costa Rica. Crisis y desafíos, San José de Costa Rica: DEI.

PANADERO, Miguel (coord). (1988), Urbanización, Subdesarrollo y Crisis en América Latina, Albacete: Seminario de Geografía.

PORTES, Alejandro. (1988), "La urbanización en América Latina en los años de crisis", en: LOMBARDI, Mario y VEGA, Danilo. (eds), Las ciudades en conflicto. Una perspectiva latinoamericana, Montevideo: CIESU.

PORTES, Alejandro y Lungo, Mario. (coords), (1992), Urbanización en Centroamérica, San José de Costa Rica: FLACSO.

PORTES, Alejandro y Lungo, Mario. (coords), (1992), Urbanización en el Caribe, San José de Costa Rica: FLACSO.

Pradilla, Emilio. (1989), "Acumulación de capital y estructura territorial en América Latina”, en: Lungo, Mario., Lo urbano: teoría y métodos, San José de Costa Rica: CSUCA.

Puncel Chornet, Alfonso (ed). (1994), Las ciudades en América Latina: problemas y oportunidades, Valencia: Universidad de Valencia.

SASSEn, Saskia. (1991), The Global City: New York, London, Tokyo, Princeton: Princeton University Press.

Sassen, Saskia. (1994), Cities in a World Economy, Thousand Oaks: Pine Forge Press.

ShORT, J. R., Kim, Y, KuUs, M. y Wells, H. (1996), "The dirty little secret of world cities research: data problems in comparative analysis", International Journal of Urban and Regional Research, Vol. 20, n. ${ }^{\circ} 4$. 
SINGER, P. (1995), "Poder público e organizaçoes populares no combate à pobreza. A experiência do governo Luiza Erundina em Sao Paulo -1989/1992-", en: Valladares, Licia y Prates Coelho, Magda, Governabilidade e Pobreza no Brasil, Río de Janeiro: Civilizaçao Brasileira.

SKLAIR, Leslie. (1991), Sociology of the Global System, Londres: Harvester Wheatsheaf.

TimberlaKe, Michael. (1985), Urbanization in the World Economy, Orlando: Academic Press.

Torres, Horacio. (1993), El Mapa Social de Buenos Aires (1940-1990), Buenos Aires: Facultad de Arquitectura, Diseño y Urbanismo (Serie Difusión).
URrutia, Víctor. (1994), "Espacio y Sociedad en Salvador de Bahía”, en: R. Villasante, Tomás. (coord), Las ciudades hablan, Caracas: Nueva Sociedad.

Valladares, Licia y Preteceille, Edmond (coords), (1990), Reestruturaçao Urbana: Tendências e Desafios, Sao Paulo: Nobel.

Valladares, Licia y Prates Coelho, Magda. (orgs) (1995), Gobernabilidade e Pobreza no Brasil, Río de Janeiro: Civilizaçao Brasileira.

Valladares, Licia y Prates Coelho, Magda. (1996), La investigación urbana en América Latina. Tendencias actuales y recomendaciones, MOST-UNESCO, Documentos de Debate, n. ${ }^{\circ} 4$.

\section{RESUMEN}

Desde los años ochenta, diversas investigaciones vienen analizando el impacto provocado por la globalización económica sobre la urbanización. También en las ciudades latinoamericanas se observan nuevas tendencias en cuanto a la estructuración del sistema territorial. El proceso de "modernización excluyente" impulsado en los últimos años, ha agravado la crisis social en las ciudades. Si en los próximos años se pretende comenzar a resolver los graves problemas urbanos existentes, debería producirse un importante giro en la orientación de las actuales políticas urbanas.

\section{ABSTRACT}

Since the eighties, different researchs have studied the economic globalization impact on the urbanization. Latinoamerican cities are subject to the new tendencies as well. "Exclusive modernization" process is making worse the urban social crisis. In order to solve the very serious urban problems, it's necessary to change the orientation of the present urban politics. 